\title{
Efecto de Tres Crioprotectores en la Criopreservación de Espermatozoides Epididimarios de Alpaca
}

\author{
Effect of Three Cryoprotectans on the Cryopreservation of Epididymal \\ Alpaca Spermatozoa
}

\author{
Marino Terreros C. ${ }^{1}$, Wilfredo Huanca L. ${ }^{1,3}$, Irma Arriaga C. ${ }^{1}$, Antonio Ampuero B. ${ }^{2}$
}

\section{Resumen}

El estudio tuvo por objetivo evaluar el efecto de tres crioprotectores: Dimetil Sulfóxido (DMSO), Etilenglicol (EG) y Glicerol (GL) sobre la criopreservación de espermatozoides epididimarios de alpaca. Se trabajó con testículos de alpacas mayores de tres años. Se separaron los epidídimos y se diseccionaron las colas. Los espermatozoides fueron recuperados con la fracción A del dilutor (leche descremada, yema de huevo y fructosa) y se emplearon las muestras con motilidad espermática igual o mayor a 50\% ( $\mathrm{n}=18$, motilidad $=69 \%$, integridad funcional de membrana espermática $=48 \%$ ). La fracción A con los espermatozoides fue refrigerada $\left(1^{\circ} \mathrm{C} / 5 \mathrm{~min}\right)$ hasta alcanzar $5^{\circ} \mathrm{C}$. Esta fracción se dividió en tres y se añadió un volumen igual de la fracción $\mathrm{B}$ conteniendo un crioprotector (DMSO: 7\%, 0.9M; EG: 7\%, 0.9M; GL: 7\%, 1.2M). Se llenaron pajillas de $0.5 \mathrm{ml}$ con la dilución final y se congelaron en nitrógeno líquido. Después de 7 días de almacenamiento, las motilidades posdescongelación fueron de 31,8 y $24 \%$ y los porcentajes de integridad funcional de la membrana espermática fueron de 28, 17 y 26\% para DMSO, EG y GL, respectivamente. Se llevó a cabo un ensayo de fecundación in vitro con los espermatozoides descongelados de los grupos DMSO y GL, donde la tasa de división a las $72 \mathrm{~h}$ posfecundación fue de 47 y $27 \%$, respectivamente, sin diferencias significativas. Los resultados sugieren que el dimetil sulfóxido y el glicerol son agentes crioprotectores más efectivos en comparación al etilenglicol.

Palabras clave: crioprotectores, criopreservación, espermatozoides epididimarios, alpaca, fecundación in vitro

\footnotetext{
${ }^{1}$ Laboratorio de Reproducción Animal, ${ }^{2}$ Laboratorio de Zootecnia y Producción Agropecuaria, Facultad de Medicina Veterinaria, Universidad Nacional Mayor de San Marcos, Lima, Perú

${ }^{3}$ E-mail: whuanca2002@yahoo.com

El financiamiento del estudio se hizo a través del Proyecto $N^{\circ} 064$ FINCyT-PIBAP 2008
}

Recibido: 5 de diciembre de 2014

Aceptado para publicación: 30 de abril de 2015 


\section{Abstract}

The study aimed to evaluate the effect of three cryoprotectants: Dimetil Sulfoxide (DMSO), Ethylene glycol (EG) and Glycerol (GL) on the cryopreservation of epididymal alpaca sperm. Testicles of alpacas older than 3 years were used. The epididymides were separated and the tails were dissected. The spermatozoa were recovered with fraction A of the extender (cow skim milk, egg yolk and fructose), selecting samples with sperm motility equal or higher than $50 \%(n=18$, motility $=69 \%$, sperm membrane functional integrity $=48 \%$ ). The fraction A with the sperm was refrigerated (cooling rate of $1{ }^{\circ} \mathrm{C} / 5$ $\min$ ) until $5^{\circ} \mathrm{C}$. The fraction was divided in three and added the same volume of fraction B with the cryoprotectant (DMSO: 7\%, 0.9M; EG: 7\%, 0.9M; GL: 7\%, 1.2M). Then, $0.5 \mathrm{ml}$ straws were filled with the final dilution and frozen in liquid nitrogen. After 7 days of storage, the post-thaw motilities were 31,8 and $24 \%$ and percentages of sperm membrane functional integrity were 28, 17 and 26\% for DMSO, EG and GL respectively. An in vitro fertilization trial was conducted with thawed sperm from groups DMSO and GL, where the cleavage rate at $72 \mathrm{~h}$ post-fertilization was 47 and $27 \%$ respectively and without statistical difference. The results suggest that dimetil sulfoxide and glycerol are more efficient cryoprotective agents in comparison to the ethylene glycol.

Key words: cryoprotectants, cryopreservation, epididymal sperm, alpaca, in vitro fertilization

\section{INTRODUCCIÓN}

Las peculiaridades y características reproductivas propias de cada especie dificultan el establecimiento de protocolos de criopreservación de semen. Estos protocolos, sin embargo, son usualmente desarrollados y validados para colectas de semen de reproductores en buenas condiciones físicas y de salud; no obstante, existen circunstancias en que por muerte accidental o por enfermedad se puede perder el material genético.

En estos casos, existe la posibilidad de recuperar los espermatozoides de los epidídimos y criopreservarlos (Martins et al., 2007). Además, en los camélidos sudamericanos, los espermatozoides epididimarios ofrecen un modelo más sencillo para establecer protocolos de congelación en comparación al semen obtenido mediante eyaculación, ya que no entran en contacto con el viscoso plasma seminal (Morton et al., 2007).
La criopreservación de espermatozoides epididimarios ha sido comprobada en varias especies; sin embargo, existe poca información en camélidos sudamericanos y aún no se ha logrado establecer un proceso adecuado y confiable. Los reportes de motilidad posdescongelación son variables, habiendo reportes de 14\% (Rodríguez, 2009), 17\% (Banda et al., 2010), 18\% (Morton et al., 2007), 20\% (Morton et al., 2010) y 33\% (Canorio, 2008).

En la criopreservación de cualquier material biológico se debe brindar las condiciones adecuadas para su supervivencia, y es allí donde los agentes crioprotectores cumplen una importante función. Los crioprotectores permeables son sustancias de bajo peso molecular que pueden pasar a través de la membrana celular, remplazando el volumen de agua intracelular evitando los daños producidos por la formación de cristales de hielo y, a su vez, manteniendo el volumen celular impidiendo el colapso celular por excesiva deshidratación (Medeiros et al., 2002; Rota et al., 2006). 
Dentro de los crioprotectores, el glicerol (GL) ha sido extensamente usado en la criopreservación de varios tipos de células, incluyendo el esperma mamífero (Rasul et al., 2007). Por otro lado, el Dimetil Sulfóxido (DMSO) es un agente crioprotector prometedor para las células, pues su bajo peso molecular $(78.13 \mathrm{~g} / \mathrm{mol})$ le permite atravesar la membrana celular de manera rápida (Ávila-Portillo et al., 2006); en tanto que el etilenglicol (EG) es un crioprotector que ha sido utilizado universalmente en la congelación de tejido ovárico y de embriones de muchas especies (Rodrigues et al., 2004), y con el semen de toro ejerce un menor efecto inhibitorio en la motilidad que el GL o el DMSO (Guthrie et al., 2002).

\section{Materiales y Métodos}

\section{Material Biológico y Dilutores}

Un total de 32 pares de testículos de alpacas mayores a 3 años de edad fueron recolectados en el camal de Pilpichaca (provincia de Huaytará, departamento de Huancavelica) entre enero y agosto de 2010. Los testículos fueron inmersos en solución tamponada para su traslado al Laboratorio de Reproducción Animal de la Facultad de Medicina Veterinaria, Universidad Nacional Mayor de San Marcos, Lima. El tiempo de transporte del material biológico fue de 8 a $10 \mathrm{~h}$, empleándose $2 \mathrm{~h}$ adicionales en obtener los espermatozoides.

La fracción A del dilutor incluyó leche fresca descremada UHT (Gloria S.A.), 5\% $\mathrm{v} / \mathrm{v}$ de yema de huevo y $4.85 \% \mathrm{p} / \mathrm{v}$ de fructosa. Se utilizó $2.5 \mathrm{ml}$ del dilutor para la recuperación de espermatozoides de los epidídimos y para su mantenimiento en refrigeración. La fracción $\mathrm{B}$ del dilutor estuvo compuesta por la fracción A y $14 \% \mathrm{v} / \mathrm{v}$ de cada crioprotector (DMSO, Sigma; EG, Merck; GL, Mallinckrodt Baker).

\section{Espermatozoides Epididimarios}

Los testículos fueron lavados con solución tamponada a $37^{\circ} \mathrm{C}$. Los epidídimos fueron removidos y desinfectados con alcohol al $70 \%$. El par de epidídimos fue considerado como una sola muestra. Se separaron las colas de ambos epidídimos y fueron diseccionadas. Se les agregó $2.5 \mathrm{ml}$ de la fracción A a $37^{\circ} \mathrm{C}$ con el fin de recuperar los espermatozoides. Luego, se recuperó la fracción A con los espermatozoides y se colocó en baño maría a $37^{\circ} \mathrm{C}$ por $5 \mathrm{~min}$. Se evaluó la calidad espermática, aceptado únicamente muestras con motilidad mayor o igual a $50 \%$. Al final, se seleccionaron 18 muestras con $68.9 \pm$ $6.1 \%$ de motilidad, lo cual corresponde al $52.3 \%$ de pares de testículos utilizados.

Para la congelación, la fracción A conteniendo los espermatozoides se dividió en tres partes para la adición de los tres crioprotectores en estudio (DMSO, EG, GL). Esta fracción se llevó a una velocidad de enfriamiento de $1{ }^{\circ} \mathrm{C}$ por cada 5 min para disminuir la temperatura de 37 a $5^{\circ} \mathrm{C}$. A esa temperatura se combinó con volúmenes iguales de la fracción $\mathrm{B}$ de cada crioprotector, obteniéndose en cada caso una concentración final de 7\% cada crioprotector del 7\%; esto es, $0.9 \mathrm{M}$ para GL, $0.9 \mathrm{M}$ para DMSO y 1.2M para EG.

La solución conteniendo los espermatozoides fue transferida a pajillas de $0.5 \mathrm{ml}$, sellándose el borde libre de cada pajilla con alcohol polivinílico para iniciar el proceso de congelación. Las pajillas fueron colocadas horizontalmente por encima de la superficie del nitrógeno líquido contenido en una caja de tecnopor para lograr su exposición a los vapores, primero a una distancia de $8 \mathrm{~cm}$ por $10 \mathrm{~min}$ y luego a $4 \mathrm{~cm}$ por $10 \mathrm{~min}$, para luego ser sumergidas en el nitrógeno. Se congelaron tres pajillas con la dilución final por muestra, dando un total de 162 pajillas (54 por tratamiento). Las pajillas quedaron en el tanque de nitrógeno líquido por siete días. 


\section{Evaluación de la Calidad Espermática}

Se hizo una primera evaluación de la calidad de los espermatozoides luego de su recuperación de los epidídimos y una segunda evaluación posterior a la congelación-descongelación de las pajillas. Para la descongelación, cada pajilla se retiró del tanque, se agitó manualmente por breves segundos y se sumergió en agua a $37{ }^{\circ} \mathrm{C}$ durante $1 \mathrm{~min}$. La pajilla fue secada y su contenido se recuperó en un vial que se llevó a baño maría a $37^{\circ} \mathrm{C}$ por $5 \mathrm{~min}$. Se evaluaron 36 pajillas por tratamiento; es decir, un total de 108 pajillas.

Motilidad espermática. Se colocaron $20 \mu \mathrm{l}$ de la muestra sobre una lámina porta-objetos temperada a $37^{\circ} \mathrm{C}$, se cubrió con una lámina cubre-objeto y se observó en el microscopio a 400x. Se determinó el porcentaje de espermatozoides mótiles sobre el total de espermatozoides observados en 10 campos de la muestra.

Integridad funcional de la membrana espermática. Se utilizó la prueba HOST (Hipoosmotic Swelling Test). Se incubó $25 \mu 1$ de la muestra en $500 \mu \mathrm{l}$ de solución hipoosmótica durante $30 \mathrm{~min}$ a $37^{\circ} \mathrm{C}$ y luego se observó una gota de la muestra al microscopio. Se evaluaron 200 espermatozoides, considerándose Host+, y por consiguiente con membrana espermática funcional, a los que mostraron una hinchazón en la parte distal de la cola espermática, y Host- (funcionalmente dañados) a aquellos que no mostraron cambio alguno en la cola espermática.

\section{Evaluación de la Capacidad Fecundante}

La capacidad fecundante de los espermatozoides de los grupos con mejor supervivencia posdescongelación (DMSO y GL) se evaluó con un ensayo de fecundación in vitro para determinar la tasa de división de los ovocitos a las $72 \mathrm{~h}$ posfecundación. Para esto, se hizo una prueba previa de maduración in vitro y un cultivo in vitro posterior con los óvulos fecundados.
Maduración in vitro. Catorce ovarios de alpacas sacrificadas en el camal de Pilpichaca fueron recolectados y llevados al laboratorio en solución salina ( $\mathrm{NaCl} 0.9 \%)$ con antibiótico antimicótico. En el laboratorio, se diseccionaron los ovarios para recuperar la mayor cantidad de complejos cúmulus-ovocitos (CCOs). Los ovocitos se seleccionaron con un estereomicroscopio, considerando la presencia de una o más capas completas de células del cúmulus no expandidas con un aspecto compacto, un citoplasma homogéneo y granulado, frente a ovocitos parcial o completamente desnudados. Se seleccionaron 30 ovocitos que fueron distribuidos entre los grupos DMSO y GL. Los ovocitos se colocaron en gotas de maduración y llevados a una estufa a $39{ }^{\circ} \mathrm{C}$ con $5 \%$ de $\mathrm{CO}_{2}$ y $20 \%$ de $\mathrm{O}_{2}$. El tiempo total de maduración fue de $40 \mathrm{~h}$.

Fecundación in vitro. El contenido de dos pajillas de la misma muestra por tratamiento y que fueron descongeladas y mantenidas a $37^{\circ} \mathrm{C}$, se colocaron en una gradiente discontinua de Percoll de 22.5/45\%, se centrifugaron por $10 \mathrm{~min}$ a $600 \mathrm{G}$ y luego por 5 min a 300 G. Finalmente, los pellets obtenidos fueron reconstituidos para la fecundación. De forma similar, después del proceso de maduración, los ovocitos fueron recuperados, colocados en gotas de fecundación y llevados a la estufa por $1 \mathrm{~h}$. Luego, se colocó $2 \mu \mathrm{l}$ de la suspensión de espermatozoides en las gotas con los ovocitos maduros. Se añadió a cada gota $2 \mu 1$ de heparina al $2 \%$ y $2 \mu 1$ de PHE ( $2 \mathrm{mM}$ penicilamina, $1 \mathrm{mM}$ hipotaurina $\mathrm{y}$ $250 \mathrm{mM}$ epinefrina). Las gotas se colocaron en la estufa por $20 \mathrm{~h}$.

Cultivo in vitro. Al finalizar el proceso de fecundación in vitro, los presuntos cigotos fueron colocados en gotas de cultivo y llevados a la estufa por $48 \mathrm{~h}$. Los embriones se evaluaron a las $72 \mathrm{~h}$ posfecundación en base a la observación de la división celular (Khatir et al., 2007). 
Cuadro 1. Efecto de los crioprotectores dimetil sulfóxido (DMSO), etilenglicol (EG) y glicerol (GL) sobre la motilidad posdescongelación en espermatozoides de alpacas

\begin{tabular}{cccc}
\hline $\begin{array}{c}\text { Motilidad inicial de } \\
\text { espermatozoides }(\%) \\
(\mathrm{n}=18)\end{array}$ & $\begin{array}{c}\text { Motilidad post-descongelación }(\%) \\
(\mathrm{n}=36)\end{array}$ & $\begin{array}{c}\text { EG } \\
(\mathrm{n}=36)\end{array}$ & $\begin{array}{c}\text { GL } \\
(\mathrm{n}=36)\end{array}$ \\
\hline $68.9 \pm 6.1$ & $31.1 \pm 8.5^{\mathrm{a}}$ & $8.3 \pm 6.9^{\mathrm{b}}$ & $23.9 \pm 8.3^{\mathrm{a}}$ \\
\hline${ }^{\mathrm{a}, \mathrm{b}}$ Superíndices diferentes indican diferencias significativas $(\mathrm{p}<0.05$ &
\end{tabular}

Cuadro 2. Efecto de los crioprotectores dimetil sulfóxido (DMSO), etilenglicol (EG) y glicerol (GL) sobre la integridad funcional de la membrana espermática posdescongelación en la alpaca

\begin{tabular}{cccc}
\hline \multirow{2}{*}{$\begin{array}{c}\text { Integridad funcional de la } \\
\text { membrana espermática inicial } \\
(\%) \\
(n=18)\end{array}$} & \multicolumn{3}{c}{$\begin{array}{c}\text { Integridad funcional de la membrana espermática post- } \\
\text { descongelación }(\%)\end{array}$} \\
\cline { 2 - 4 } & $\begin{array}{c}\text { DMSO } \\
(\mathrm{n}=36)\end{array}$ & $\begin{array}{c}\mathrm{EG} \\
(\mathrm{n}=36)\end{array}$ & $\begin{array}{c}\text { GL } \\
(\mathrm{n}=36)\end{array}$ \\
\hline $53.9 \pm 8.5$ & $28.7 \pm 5.8^{\mathrm{a}}$ & $17.8 \pm 7.0^{\mathrm{b}}$ & $26.4 \pm 8.3^{\mathrm{a}}$ \\
\hline${ }^{\mathrm{a}, \mathrm{b}}$ Superíndices diferentes indican diferencias significativas $(\mathrm{p}<0.05)$ &
\end{tabular}

\section{Análisis Estadístico}

Los porcentajes de motilidad espermática y de los espermatozoides HOST + se transformaron a ángulos (ángulo = arcoseno «x) para acercar los valores a la distribución normal. Para determinar la existencia de diferencias estadísticas se utilizó la prueba de análisis de varianza (ANOVA), así como la prueba postest de Tukey para determinar diferencias estadísticas entre grupos. Los datos de la fecundación in vitro fueron analizados con la prueba de Chi cuadrado.

\section{Resultados}

Los espermatozoides congelados con DMSO fueron los que obtuvieron mejor motilidad posdescongelación $(31.1 \pm 8.5 \%)$ seguidos de aquellos congelados con glicerol $(23.9 \pm 8.3 \%)$, habiendo diferencias signifi- cativas entre estos dos grupos con el grupo de etilenglicol (Cuadro 1). La integridad funcional inicial de la membrana espermática fue de $53.9 \pm 8.5 \%$, mientras que los resultados luego de la descongelación siguieron el mismo patrón que la motilidad (Cuadro 2).

En relación a la fecundación in vitro, la tasa de división de los 15 ovocitos a las $72 \mathrm{~h}$ posfecundación fue estadísticamente similar entre el grupo DMSO (46.6\%) y el grupo GL $(26.6 \%)$.

\section{Discusión}

La criopreservación de semen de camélidos ha mostrado usualmente resultados bastante variables. En el presente estudio, las dos mejores motilidades posdescongelación fueron de 24 y $31 \%$; lo que si bien no son valores altos, cabe señalar que la via- 
bilidad de los espermatozoides disminuye entre un 40 a $50 \%$ durante el proceso de criopreservación (Watson, 2000), e incluso los mejores protocolos de criopreservación no garantizan una alta supervivencia de los espermatozoides (Medeiros et al., 2002). Es así, que los valores obtenidos son cercanos a lo teóricamente esperado considerando que la motilidad inicial fue de $69 \%$.

El 24\% de motilidad posdescongelación obtenida con el empleo de glicerol ha sido uno de los mejores resultados reportados con este crioprotector en espermatozoides epididimarios, toda vez que Morton et al. (2007), Banda et al. (2010) y Morton et al. (2010) reportan motilidades de 18, 14 y $20 \%$, respectivamente. Este resultado se debería a la acción conjunta del crioprotector con los componentes del dilutor, ya que tanto la yema de huevo como las proteínas de la leche ejercen funciones crioprotectoras que ayudan al glicerol. Esto también se evidencia en la aproximación a los resultados de Morton et al. $(2007,2010)$, quienes usaron un dilutor a base a lactosa, mientras que Banda et al. (2010) emplearon un dilutor en base a Tris.

En la mayoría de estudios en criopreservación de semen de camélidos se ha utilizado altas concentraciones de GL (6$7 \%$ ) como en el presente estudio, mientras que los trabajos de Morton et al $(2007,2010)$ usaron concentraciones bajas (3-4\%), las que también parecen ser adecuadas para la criopreservación. Se requiere mayores estudios para establecer la concentración adecuada de glicerol en la criopreservación de espermatozoides epididimarios de alpaca.

La mayor supervivencia espermática con el uso de dimetil sulfóxido se debería a la rápida penetración en las células erspermáticas del DMSO por su menor peso molecular $(78.13 \mathrm{~g} / \mathrm{mol})$ en comparación al glicerol $(92.09 \mathrm{~g} / \mathrm{mol})$, lo que favorecería su acción protectora. El $31 \%$ de motilidad posdescongelación obtenido con este crioprotector fue similar al $33 \%$ obtenido por Canorio (2008), quienes emplearon un dilutor a base de citrato, y cuya molaridad final del crioprotector fue $0.25 \mathrm{M}$, en comparación con el $0.9 \mathrm{M}$ del presente estudio.

La baja eficiencia del etilenglicol difiere de los resultados de Banda et al. (2010), quienes obtuvieron una motilidad de $17 \%$ con este crioprotector. Esta diferencia se debería a la mayor susceptibilidad de los espermatozoides de alpaca a los efectos tóxicos de concentraciones altas de EG. La molaridad empleada en el presente trabajo fue de $1.2 \mathrm{M}$, mientras que en el otro estudio fue de $0.2 \mathrm{M}$.

Los resultados de integridad funcional de la membrana espermática reafirma el buen desempeño de DMSO y Gl como crioprotectores.

El presente trabajo es la primera experiencia reportada de fecundación in vitro en alpacas usando espermatozoides epididimarios poscriopreservación. No obstante, la cantidad de ovocitos procesados fue pequeña (15 por grupo) en comparación a otros trabajos de fecundación en camélidos (Del Campo et al., 1994; Khatir y Anouassi, 2006) donde se emplearon 234 y 664 ovocitos, respectivamente. Sin embargo, estos resultados son auspiciosos ya que se ha comprobado la posibilidad de fecundar in vitro ovocitos de alpaca con este tipo de espermatozoides.

\section{Literatura Citada}

1. Ávila-Portillo LM, Madero JI, López C, León MF, Acosta L, Gómez C, Delgado LG et al. 2006. Fundamentos de criopreservación. Rev Colombiana Obst Ginecol 57: 291-300.

2. Banda RJ, Evangelista VS, Ruiz GL, Sandoval MR, Rodriguez LC, Valdivia CM, Santiani AA. 2010. Efecto de dilutores en base a Tris, Tes y Leche descremada en la criopreservación de espermatozoides obtenidos del epididimo de alpaca. Rev Inv Vet, Perú 21: 145153. doi: 10.15381/rivep.v21i2.129 
3. Canorio PN. 2008. Criocapacitación del espermatozoide de alpaca (Lama pacos). Tesis de Magíster. Lima: Universidad Nacional Mayor de San Marcos. $102 \mathrm{p}$.

4. Del Campo MR, Del Campo CH, Donoso MX, Berland M, Mapletoft RJ. 1994. In vitro fertilization and development of llama (Lama glama) occytes using epididymal spermatozoa and oviductal cell co-culture. Theriogenology 41: 1219-1229.

5. Guthrie HD, Liu J, Critser JK. 2002. Osmotic tolerance limits and effects of cryoprotectans on motility of bovine spermatozoa. Biol Reprod 67: 1811-1816.

6. Khatir H, Anouassi A. 2006. The first dromedary (Camelus dromedarius) offspring obtained from in vitro matured, in vitro fertilized and in vitro cultured abattoir-derived oocytes. Theriogenology 65: 1727-1736.

7. Khatir H, Anouassi A, Tibary A. 2007. Effect of follicular size on in vitro developmental competence of oocytes and viability of embryos after transfer in the dromedary (Camelus dromedarius). Anim Reprod Sci 99: 413-420. doi: 10.1016/j.anireprosci.2006.06.015

8. Martins CF, Rumpf R, Pereira DC, Dode MN. 2007. Cryopreservation of epididymal bovine spermatozoa from dead animals and its uses in vitro embryo production. Anim Reprod Sci 101: 326331. doi: 10.1016/j.anireprosci. 2007.01.018

9. Mazur P, Leibo SP, Seidel GE. 2008. Cryopreservation of the germplasm of animals used in biological and medical research: Importance, impact, status, and future directions. Biol Reprod 78: 2-12. doi: 10.1095/biolreprod.107.064113

10. Medeiros CM, Forel F, Oliveira AT, Rodrigues JL. 2002. Current status of sperm cryopreservation: why isn't it better? Theriogenology 57: 327-344. doi: 10.1016/S0093-691X(01)00674-4

11. Morton KM, Bathgate R, Evans $G$, Maxwell CWM. 2007. Cryopreservation of epididymal alpaca (Vicugna pacos) sperm: a comparison of citrate-, Tris- and lactose-based diluents and pellets and straws. Reprod Fertil Dev 19: 792-796. doi: 10.1071/RD07049

12. Morton KM, Evans G, Mawwell WMC. 2010. Effect of glycerol concentration, Equex STM ${ }^{\circledR}$ supplementation and liquid storage prior to freezing on the motility and acrosome integrity of frozen-thawed epididymal alpaca (Vicugna pacos) sperm. Theriogenology 74: 311-316. doi: $10.1016 / \mathrm{j}$.theriogenology. 2010.02 .015

13. Rasul Z, Ahmed N, Anzar M. 2007. Antagonist effect of DMSO on the cryoprotection ability of glycerol during cryopreservation of buffalo sperm. Theriogenology 68: 813-819. doi: 10.1016/j.theriogenology.2007.06.014

14. Rodrigues APR, Amorim CA, Costa SHF, Matos MHT, Santos RR, Lucci CM, Báo SN, et al. 2004. Cryopreservation of caprine ovarian tissue using glycerol and ethylene glycol. Theriogenology 61: 1009-1024.

15. Rodríguez L. 2009. Efecto del plasma seminal sobre la supervivencia de espermatozoides criopreservados de alpaca (Vicugna pacos). Tesis de Magister. Lima: Universidad Nacional Mayor de San Marcos. 72 p.

16. Rota A, Milani C, Cabianca G, Martini M. 2006. Comparison between glycerol and ethyleneglycol for dog semen cryopreservation. Theriogenology 65 : 1848-1858. doi: 10.1016/j.theriogenology. 2005.10.015

17. Watson PF. 2000. The causes of reduced fertility with cryopreserved semen. Anim Reprod Sci 60: 481-492. doi: 10.1016/S0378-4320(00)00099-3 